www.jmscr.igmpublication.org Impact Factor 5.244

Index Copernicus Value: 5.88 ISSN (e)-2347-176x ISSN (p) 2455-0450 crossref DOI:_http://dx.doi.org/10.18535/jmscr/v4i6.21

\title{
Lipid Profile in Type 2 Diabetes Mellitus with and Without Subclinical Hypothyroidism
}

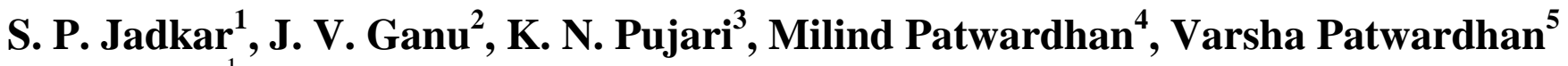 \\ ${ }^{1} \mathrm{Ph}$ D Student, Department of Biochemistry, Govt. Medical College, Miraj \\ ${ }^{2}$ Associate Professor, Department of Biochemistry, Govt. Medical College, Miraj \\ ${ }^{3}$ Associate Professor, Department of Biochemistry, R.C.S.M. Govt. Medical College, Kolhapur \\ ${ }^{4}$ Endocrinologist, Patwardhan's Endocrine research centre, Miraj \\ ${ }^{5}$ Pathologist, Patwardhan's Endocrine research centre, Miraj \\ Corresponding Author \\ Dr Karan Ningappa Pujari \\ Associate professor, Department of Biochemistry, \\ R.C.S.M. Govt. Medical College, Kolhapur (Maharashtra, India). \\ Email:pujari_karyappa@yahoo.in
}

\begin{abstract}
In clinical practice, Diabetes Mellitus is the most common endocrine disorder. The prevalence of thyroid disorders is higher in diabetic patients as compared to general population and the most common disorder being subclinical hypothyroidism. Subclinical hypothyroidism has been claimed to be a risk factor for coronary artery disease, peripheral vascular disease and various biochemical abnormalities including dyslipidemia.

The present study was planned to measure serum lipids such as total cholesterol, triglycerides, low density lipoprotein- cholesterol and high density lipoprotein- cholesterol in type 2 diabetics with and without subclinical hypothyroidism. Study includes 200 patients of type 2 diabetics with subclinical hypothyroidism and 200 type 2 diabetics without subclinical hypothyroidism. The data were evaluated statistically. We found significantly increase in total cholesterol, triglycerides and low density lipoprotein- cholesterol $(p<0.001)$ and significantly decreased high density lipoprotein-cholesterol $(p<0.001)$ in type 2 diabetes mellitus with subclinical hypothyroidism before therapy as compared to type 2 diabetes mellitus without subclinical hypothyroidism.

After L-thyroxine therapy we observed highly significant reduction in total cholesterol, triglycerides and low density lipoprotein- cholesterol $(p<0.001)$ and significantly increase in high density lipoproteincholesterol $(p<0.001)$ in type 2 diabetes mellitus with subclinical hypothyroidism as compared to before therapy.

Subclinical hypothyroidism is associated with atherogenic serum lipid profile pattern. Such pattern may increase the risk of atherosclerosis and CVD. L-thyroxine is of utmost importance to reduce or prevent the risk of development of atherosclerosis and CVD in type 2 DM with SCH patients.

Key Words: Diabetes Mellitus, Subclinical hypothyroidism, Cardiovascular disease, Lipid profile.
\end{abstract}




\section{Introduction}

In clinical practice, Diabetes Mellitus (DM) is the most common endocrine disorder. India has become the "diabetes capital" of the world with over three crore affected patients (1). The prevalence of thyroid disorders is higher in diabetic patients as compared to general population. In type 2 diabetes mellitus the prevalence of thyroid disorder has been found to be $31 \%$, the most common disorder being subclinical hypothyroidism $(\mathrm{SCH})^{(2)}$.

$\mathrm{SCH}$ is the condition characterized by elevated thyroid stimulating hormone (TSH) levels with normal serum free thyroxine (T4) and triiodothyronine (T3) levels (3). Recently, subclinical hypothyroidism has been claimed to be a risk factor for coronary artery disease, peripheral vascular disease and various biochemical abnormalities including dyslipidemia ${ }^{(4)}$.

Lipid disorders are common in diabetes mellitus and play crucial roles in the development of diabetic cardiovascular complications. Presence of thyroid dysfunction may affect diabetic control and thyroid disorders are known to influence on lipid metabolism ${ }^{(5)}$.

However, there is a lack of consistent data on whether and to what extent does $\mathrm{SCH}$ affect lipid profile ${ }^{(4)}$. Moreover, there are controversial debates regarding the effect of $\mathrm{SCH}$ therapy with levothyroxine on serum lipids ${ }^{(6)}$.

Therefore, we assessed the effect of levothyroxine (L-thyroxine) treatment on lipid profile in diabetics with $\mathrm{SCH}$.

\section{Materials and methods}

The present study was carried out in the Department of Biochemistry, Government medical college and Patwardhan's Endocrine Research Center, Miraj. The study was approved by the ethics committee of Government medical college, Miraj (Maharashtra, India).

\section{Selection of the study subjects}

Study included total 400 subjects and grouped in to two groups.
Group I: includes 200 patients suffering from type 2 diabetes mellitus with subclinical hypothyroidism (age 40-60 years) diagnosed by endocrinologist based on WHO criteria for diabetes mellitus and normal T3, T4 with TSH level > $4.5 \mu \mathrm{IU} / 1$ for $\mathrm{SCH}$ at Patwardhan's Endocrine Research Center, Miraj.

L-Thyroxine therapy of $12.5 \mu \mathrm{g}$ per day was prescribed to diabetics with subclinical hypothyroidism patients for 3 months by endocrinologist.

Pretherapy and post therapy blood samples were collected for various biochemical investigations. Internal comparison of pre and post therapy results was done.

Group II: includes 200 type 2 Diabetes Mellitus patients without subclinical hypothyroidism (age 40-60 years) were diagnosed by endocrinologist based on WHO criteria and having normal T3, T4 and TSH levels.

Subjects having history or symptoms of cardiovascular diseases, receiving other medications that alter thyroid functions and lipid levels were excluded from the study.

\section{Sample (Blood) collection}

An informed consent was obtained from participants after complete explanation of procedure. Every patient was advised for at least 12-14 hours overnight fast. About $3 \mathrm{ml}$ venous blood was collected in plain blub taking aseptic precautions and used for lipid parameter investigations.

After two hours of collection, samples were centrifuged at 3000 R.P.M. for 10 minutes, clear and un-hemolysed serum was separated and used for estimation of Total cholesterol (TC), HDL cholesterol (HDL-C), LDL cholesterol (LDL-C) and triglycerides (TG).

Total cholesterol estimated by Enzymaticcolorimetric (Trinder - Endpoint) (7,8), Triglycerides by Enzymatic-colorimetric (Endpoint) method ${ }^{(8-10)}$ and direct High density lipoprotein cholesterol (HDL-C) ${ }^{(11)}$ and direct Low density lipoprotein cholesterol (LDL-C) ${ }^{(12)}$ 


\section{JMSCR Vol||04||Issue||06||Page 10807-10812||June}

on Selectra Junior analyzer by using Merck specialties private limited kit. The data was analyzed by student's ' $t$ ', ' $z$ ' test and Karl Pearson's correlation coefficient. $\mathrm{P}$ values $<0.001$ were considered significant.

\section{Results}

Table no 1 show lipid parameters in subjects. We found highly significant increase $(\mathrm{p}<0.001)$ in total cholesterol, triacylglycerol, LDL-C and highly significant decrease $(\mathrm{p}<0.001)$ in HDL-C in type $2 \mathrm{DM}$ with $\mathrm{SCH}$ patients as compared to type $2 \mathrm{DM}$ without $\mathrm{SCH}$.

Comparison of lipid parameters in type $2 \mathrm{DM}$ with $\mathrm{SCH}$ patients before and after therapy is given in table no 2. We observed highly significant reduction in total cholesterol, TG and LDL-C and highly significant increase in HDL-C after therapy as compared to before therapy.

We found only significant correlation of TSH with TG before treatment $(\mathrm{p}<0.05)($ table no.3).

Table No 1: Serum lipid parameters in subjects

\begin{tabular}{|c|c|c|c|}
\hline Parameters & $\begin{array}{l}\text { Type } 2 \text { DM } \\
(n=200)\end{array}$ & $\begin{array}{l}\text { Type 2 DM with SCH } \\
\text { before } \\
(\mathrm{n}=200)\end{array}$ & $P$ value \\
\hline Total cholesterol (mg/dl) & $227 \pm 19.683$ & $237.621 \pm 20.054$ & 0.001 \\
\hline Triglycerides (mg/dl) & $184.905 \pm 26.139$ & $223.317 \pm 23.045$ & 0.001 \\
\hline HDL (mg/dl) & $23.625 \pm 3.672$ & $21.25 \pm 4.350$ & 0.001 \\
\hline LDL (mg/dl) & $104.2 \pm 18.48$ & $123.293 \pm 17.432$ & 0.001 \\
\hline
\end{tabular}

Table No 2: Comparison of lipid parameters in patients before and after treatment

\begin{tabular}{|l|l|l|l|l|}
\hline Parameters & $\begin{array}{l}\text { Type 2 DM with SCH } \\
\text { Before treatment } \\
(\mathbf{n = 2 0 0})\end{array}$ & $\begin{array}{l}\text { Type 2 DM with SCH } \\
\text { After treatment (n=200) }\end{array}$ & t value & P value \\
\hline Total cholesterol (mg/dl) & $237.621 \pm 20.0543$ & $219.482 \pm 16.643$ & 15.39 & 0.001 \\
\hline Triglycerides (mg/dl) & $223.317 \pm 23.045$ & $210.169 \pm 23.615$ & 20.87 & 0.001 \\
\hline HDL (mg/dl) & $21.25 \pm 4.350$ & $24.04 \pm 4.457$ & -18.60 & 0.001 \\
\hline LDL (mg/dl) & $123.293 \pm 17.432$ & $115.737 \pm 17.191$ & 17.31 & 0.001 \\
\hline
\end{tabular}

Table No 3: Correlation between TSH level and lipid parameters of type 2 DM with SCH patients before treatment

\begin{tabular}{|l|l|l|l|}
\hline & Lipid parameters & R & P \\
\hline TSH in $(\boldsymbol{\mu I U} / \mathrm{l})$ & TC $(\mathrm{mg} / \mathrm{dl})$ & -0.072 & 0.311 \\
\hline & TG $(\mathrm{mg} / \mathrm{dl})$ & $-0.139 *$ & 0.05 \\
\hline & HDL-C $(\mathrm{mg} / \mathrm{dl})$ & 0.036 & 0.612 \\
\hline & LDL-C $(\mathrm{mg} / \mathrm{dl})$ & 0.036 & 0.615 \\
\hline & &
\end{tabular}

Table No 4: Correlation between TSH level and lipid parameters of type 2 DM with SCH patients after treatment

\begin{tabular}{|l|l|l|l|}
\hline & Lipid parameters & $\mathbf{R}$ & $\mathbf{P}$ \\
\hline TSH in $(\boldsymbol{\mu} \mathbf{I U} / \mathrm{l})$ & TC $(\mathrm{mg} / \mathrm{dl})$ & -0.077 & 0.280 \\
\hline & TG $(\mathrm{mg} / \mathrm{dl})$ & 0.086 & 0.225 \\
\hline & HDL-C $(\mathrm{mg} / \mathrm{dl})$ & 0.036 & 0.608 \\
\hline & LDL-C $(\mathrm{mg} / \mathrm{dl})$ & 0.066 & 0.353 \\
\hline
\end{tabular}

\section{Discussion}

In the present study we have demonstrated that patients with type $2 \mathrm{DM}$ with $\mathrm{SCH}$ had altered lipid profile when compared to type $2 \mathrm{DM}$ without $\mathrm{SCH}$. This indicates lipid abnormalities in type 2
DM with SCH. High level of TSH may associated with deleterious changes in serum lipids such as total cholesterol, low density lipoprotein cholesterol, high density lipoprotein cholesterol and triglycerides. 
Study of Shivleela Biradar et al ${ }^{(4)}$ found similar findings in $\mathrm{SCH}$, which supports our study but patients included in their study were non-diabetic. Another study of Sarika Singh et al (13, 14) observed significant increase in TC, TG LDL-C and non-significant decrease in HDL-C in $\mathrm{SCH}$ patients without DM compared to controls.

Dyslipidemia is a common finding in patients with thyroid disease, explained by the adverse effects of thyroid hormones in almost all steps of lipid metabolism ${ }^{(4,14)}$. SCH by different mechanisms can be associated with lipid alterations, mainly total and LDL-C and less often HDL-C, TG, and Lp (a), which may increase the risk of heart diseases ${ }^{(14)}$.

Hypercholesterolemia is a common feature in hypothyroidism because thyroid hormones upregulate LDL-receptor expression ${ }^{(15)}$. The reported mechanisms for the development of hypercholesterolemia and an increase in low density lipoproteins in hypothyroidism include decreased fractional clearance of LDL by a reduced number and reduced activity of LDL receptors in the liver ${ }^{(4,13)}$.

In vivo and in vitro studies of Wei Zhang et al ${ }^{(16)}$ showed the presence of TSH receptor on liver cells. TSH itself upregulates the expression of HMG-COA -reductase by acting on a TSH receptor in hepatocyte membrane and therefore promotes cholesterol synthesis in liver ${ }^{(17)}$. Such mechanisms may initiate lipid abnormalities in $\mathrm{SCH}$.

Increase in serum TSH levels may decrease the activities of hepatic lipase, lecithin cholesterol acyl transferase and ATP binding cassette transporter resulting in reduction in HDL-C levels (4)

High level of TG may be due decreased activity of lipoprotein lipase enzyme ${ }^{(4)}$ and poor clearance of endogenous and exogenous triglycerides from circulation in subclinical hypothyroidism (13). Thyroid hormones affect lipoprotein lipase activity and which regulates hydrolysis of TG in VLDL and chylomicrons into fatty acids and glycerol. In hypothyroidism, lipoprotein lipase activity in the adipose tissue has been found normal or decreased in addition to decreased hepatic lipase activity resulting in normal or high levels of $\mathrm{TG}^{(14)}$.

TG- rich lipoproteins such as chylomicron remnants and VLDL remnants still play an important role in atherogenesis. These remnants are taken up by macrophages in the arterial walls to produce foam cells and thus may be a risk factor for atherosclerosis in $\mathrm{SCH}^{(4)}$.

\section{Effect of thyroxine therapy on lipid profile}

After therapy in our study, we found highly significant decrease in TC, TG, LDL-C and highly significant increase in HDL-C values compared to before therapy in type 2DM with $\mathrm{SCH}$. This may indicate reduction in the cardiovascular risk factors.

Similarly Sarika Singh et al ${ }^{(14)}$ and Amit Saxena et al ${ }^{(18)}$ found significant decrease in $\mathrm{TC}, \mathrm{TG}$, LDL-C and significant increase in HDL-C levels after L-thyroxine therapy as compared to before therapy in non-diabetic $\mathrm{SCH}$ patients ${ }^{(14)}$.

After L-thyroxine therapy in $\mathrm{SCH}$ serum $\mathrm{TSH}$ concentration decreases as serum $\mathrm{T} 4$ concentration rises that may lead to increased conversion of cholesterol into bile acids in liver, activity of lipoprotein lipase may be elevated, increased synthesis of proteins required for HDL synthesis and more expression and synthesis of LDL receptors. All these mechanisms may results in reduction in the concentration of $\mathrm{TC}, \mathrm{TG}$ and LDL-C and increased concentration of HDL-C in type $2 \mathrm{DM}$ with $\mathrm{SCH}$ after therapy.

\section{Conclusion}

$\mathrm{SCH}$ is associated with atherogenic serum lipid profile pattern. Such pattern (atherogenic lipid profile that is TC, HDL-C, TG, and LDL-C) may increase the risk of atherosclerosis and CVD. Therefore there is a potential association between $\mathrm{SCH}$ and atherosclerosis. So assessment, monitoring of serum lipid profile and treatment of L-thyroxine is of utmost importance to reduce or prevent the risk of development of atherosclerosis and CVD in type $2 \mathrm{DM}$ with $\mathrm{SCH}$ patients. 


\section{References}

1. Vinu Vij, Pallavi Chitnis, Vijay Kumar Gupta. Evaluation of thyroid dysfunction among type II diabetic patients. International Journal of Pharmacy and Biological Sciences Oct- Dec 2012; 2(4): 150-155.

2. Satvic C. Manjunath, Vishwanath Krishnamurthy, Balaraj K. Puttaswamy, Srinivasa Prabhu, Poornachandra M. Vishwanathaiah. Prevalence of subclinical thyroid disorders in type 2 diabetes mellitus. International Journal of Medicine and Public Health Oct- Dec 2013: 3(4):330-334.

3. S. Senthikumaran, V. Sathyaprakash and A. Sundhararajan. A study on prevalence and distribution of subclinical hypothyroidism in rural women. Scholars journal of Applied Medical Sciences 2015; 3(1D):287-290.

4. DF 40. Shivaleela M. Biradar and Santosh R. Patil. Study of lipid profile in subclinical hypothyroidism. International Journal of Pharma and Bio Sciences july 2015; 6(3):B1-6.

5. Makandar Asmabi, Sonagra Amit D, Biradar Shivaleela M and Quadri Shireen Swaliha. Effect of thyroid dysfunction on metabolic response in type 2 diabetic patients. Unique Journal of Medical and Dental Sciences 2015; 3(1):65-69.

6. Karoon Shahebrahimi, Rozita Naseri, Tahereh Sadat Kalantarian, Mehrali Rahimi, Farid Najafi, Katayoun Derakhshandeh, Azam Sharifi. Effects of levothyroxine treatment on lipid profile in subclinical hypothyroidism: a randomized clinical trial. Galen Medical Journal 2015; 4(2):72-77.

7. Rifai N.,et al.,Lipids, Lipoproteins, and Apolipoproteins. Teitz Fundamentals of Clinical chemistry, 5th Ed, Burtis, C.A. \& Ashwood, E.R. by W. B. Saunders Philadelphia USA), 2001,463.
8. Naito, H K, Coronary Artery Disease and Disorders of Lipid Metabolism. Clinical Chemistry: Theory, Analysis, Correlation, 4th Ed., Kaplan, L.A., Pesce, A J., Kazmierczak, S C by Mosby, Inc. eds. St Louis USA, 2003,603.

9. Fossati P, Prencipe L. Serum triglycerides determined colorimetrically with enzyme that produces hydrogen peroxide. Clin Chem, 1982, 28:2077.

10. Tietz NW. Clinical guide to laboratory tests, 3rd edition by Saunders Philadelphia USA), 1995,610.

11. Dominiczak M, McNamara J. The system of cardiovascular prevention: in 103-125; Nauk M, Wiebe D, Warnick G. Measurement of high density lipoprotein cholesterol. 221-244. In: handbook of lipoprotein testing (eds. Rifai, Warnick and Dominiczak), 2nd edition.

12. Hongbing Xiao. Method and composition for determining low density lipoprotein cholesterol. Chinese Patent CN 1379234A (2002).

13. D. F. 32. Sarika Singh and Purnima Dey Sarkar. Lipids, oxidative stress and new emerging cardiac risk markers in subclinical hypothyroidism: before and after treatment. Journal of Pharmaceutical and Biological Sciences 2013; 1(5):61-65.

14. D.F. 36. Sarika Singh and Purnima Dey Sarkar. Serum lipids, tHcy, hs-CRP, MDA and PON-1 levels in $\mathrm{SCH}$ and overt hypothyroidism: effect of treatment. Acta Biomed 2014; 85(2):127-134.

15. D.F. 35. Adriana Santi, Marta M.M.F. Duarte, Charlene C. de Menezes and Vania Lucia Loro. Association of lipids with oxidative stress biomarkers in subclinical hypothyroidism. International journal of Endocrinology 2012; Article ID 856357:1-7.

16. D. F. 37. Wei Zhang, Li-min Tian, Yong Han, Hong-yan Ma, Lai-cheng Wang, Jun Guo, Ling Gao and Jia-Jun Zhao. Presence 
of thyrotropin receptor in hepatocytes: not a case of illegitimate transcription. J Cell Mol Med 2009; 13(11-12):4636-4642.

17. D.F. 38. Furong Wang, Yinyin Tan, Chenggang Wang, Xu Zhang, Yuanfei Zhao, Xinhong Song, Bingchang Zhang, Qingbo Guan, Jin Xu, Ji Zhang, Dongzhi Zhang, Haiyan Lin, Chunxiao $\mathrm{Yu}$ and Jiajun Zhao. Thyroid stimulating hormone levels within the reference range are associated with serum lipid profiles independent of thyroid hormone. J Clin Endocrinol Metab 2012; 97:2724-2731.

18. D. F. 39. Amit Saxena, Pragati Kapoor, Shikha Saxena and A K Kapoor. Effect of levothyroxine therapy on dyslipidemia in hypothyroid patients. Internet Journal of medical update, July 2013; 8(2):39-49. 